\section{Für eine Theorie der Gentrifizierung}

,Für eine Theorie der

Gentrifizierung'

Kommentare von: Moritz Ege, Laura Calbet, Jens Dangschat, Laura Nkula-Wenz, Lisa Vollmer, Andrej Holm

Neil Smith

Gentrifizierung wird vorrangig unter Verweis auf Konsumentensouveränität erklärt, aber Daten zur Zahl der Vorstädter, die in die Stadt zurückziehen, werfen Zweifel an dieser Hypothese auf. Tatsächlich ist Gentrifizierung ein erwartbares Ergebnis der weitgehend ungehinderten Funktionsweise des Grundstücks- und Wohnungsmarktes. Die wirtschaftliche Entwertung des in innerstädtische Quartiere aus dem 19. Jahrhundert investierten Kapitals bei gleichzeitigem Anstieg der potentiellen Höhe der Grundrente eröffnet die Möglichkeit profitabler Stadterneuerung. Auch wenn die deutlich sichtbaren sozialen Merkmale heruntergekommener Quartiere abschreckend wirken mögen, können die hintergründigen ökonomischen Merkmale durchaus für eine Sanierung sprechen. Ob Gentrifizierung eine fundamentale Neuordnung des urbanen Raums bedeutet, hängt nicht davon ab, wo die neuen Bewohner herkommen, sondern davon, wie viel Produktivkapital aus dem suburbanen Raum in die Stadt zurückkehrt.

An English abstract can be found at the end of the document.

Nach einer Phase des anhaltenden Niedergangs erleben viele amerikanische Städte die Gentrifizierung ausgewählter innerstädtischer Quartiere.[1] Erste Anzeichen der Wiederbelebung während der 1950er Jahre intensivierten sich in den 1960er Jahren und waren bis zu den 1970er Jahren zu einer großflächigen Gentrifizierungsbewegung geworden, die die Mehrzahl der älteren Städte des Landes betraf.[2] Eine neuere Untersuchung des Urban Land Institute (1976) lässt darauf schließen, dass in annähernd der Hälfte der 260 Städte mit über 50.00o Einwohnern[3] innerstädtische Gebiete saniert werden. Obwohl Gentrifizierung im Vergleich zu Neubau landesweit nur einen Bruchteil der Wohnungsbautätigkeit ausmacht, ist der Prozess in den älteren Städten des Nordostens (aber nicht nur dort) von großer Bedeutung.

Im selben Maße wie der Gentrifizierungsprozess selbst florierte auch die Literatur zum Thema, die sich zumeist mit dem aktuellen Geschehen oder mit seinen Auswirkungen befasst: mit den sozioökonomischen und kulturellen Merkmalen der Zugezogenen, mit Verdrängung, mit der Rolle der Bundespolitik bei Stadterneuerungsmaßnahmen, dem Nutzen der Gentrifizierung für die Stadt sowie der Schaffung oder Zerstörung von Gemeinschaft. Wenig wurde hingegen unternommen, um den Prozess historisch zu erklären, um statt der Auswirkungen eher die Ursachen zu ergründen. 
Stattdessen bleiben Begründungen vielfach unhinterfragt. Hier lassen sich zwei Kategorien unterscheiden, eine kulturelle und eine ökonomische.

- Kulturelle Erklärungsansätze. Eine bei Theoretikern des city revivals gängige Vorstellung ist, dass junge, in der Regel berufstätige Mittelschichtangehörige ihren Lebensstil geändert haben. Gregory Lipton zufolge hat dieser Wandel ein hinreichendes Ausmaß erreicht, um „die relative Attraktivität des Einfamilienhauses am Stadtrand zu mindern“ (1977: 146) [4]. Durch den Trend zu weniger Kindern, höherem Heiratsalter und einer rasant ansteigenden Scheidungsrate tauschen junge Immobilienkäufer somit den glanzlos gewordenen Traum ihrer Eltern gegen einen neuen ein, der eher urban als suburban definiert ist. Andere Forscher betonen die Suche nach spezifisch ausgeprägten sozialen Gemeinschaften als einem der individuellen Selbstentfaltung zuträglichen Umfeld (Winters 1978), während wieder andere auf dieser Grundlage eine allgemeiner angelegte Argumentation entwickeln. In zeitgenössischen ,postindustriellen Städten' werden David Ley zufolge Blue-Collar-Tätigkeiten in der Produktion von White-Collar-Tätigkeiten im Dienstleistungsbereich abgelöst, wodurch sich der Fokus weg von der Arbeit hin zu Konsum und den Annehmlichkeiten des Lebens verschiebt. Nun geben Konsummuster die Produktionsmuster vor: „[E]ntscheidungsleitend für die Nutzung innerstädtischer Flächen sind nicht produktions-, sondern konsumorientierte Werthaltungen." (Ley 1978: 11) Der Wiederaufstieg der Innenstadt ist ein Beispiel für diese neue Gewichtung des Konsums.

- Ökonomische Erklärungsansätze. Angesichts kontinuierlich steigender Kosten für Wohnungsneubau und dessen zunehmender Entfernung vom Stadtzentrum gilt die Sanierung von Gebäuden in Innenstadt- und Zentrumslage als wirtschaftlich rentabler. Erwerb und Sanierung alter, aber in der Bausubstanz intakter Immobilien können kostengünstiger sein als der Kauf eines vergleichbaren neuen Hauses. Darüber hinaus werden in der Forschung vielfach die hohen Pendelkosten - höhere Benzinkosten für Privatfahrzeuge und steigende Tarife im öffentlichen Verkehr - und die wirtschaftlichen Vorteile der Nähe zum Arbeitsplatz betont.

Diese gängigen Hypothesen schließen sich gegenseitig keineswegs aus. Sie werden häufig zusammen vorgebracht und nehmen in einer wichtigen Hinsicht dieselbe Perspektive ein - betont werden Konsumentenpräferenzen sowie die Restriktionen, denen die Verwirklichung dieserVorlieben unterliegt. Diese Sichtweise deckt sich weitestgehend mit dem Korpus neoklassischer Theorien über Bodennutzung zu Wohnzwecken (Alonso 1964; Muth 1969; Mills 1972). Laut neoklassischer Theorie spiegelt Suburbanisierung räumliche Präferenzen und die gestiegene Zahlungsfähigkeit hierfür, nachdem sich transportbezogene und andere Restriktionen reduziert haben. Entsprechend wird Gentrifizierung als das Ergebnis eines Wandels der Präferenzen und/ oder der Restriktionen erklärt, die darüber entscheiden, welche Präferenzen verwirklicht werden können. Folglich wird der Prozess in den Medien wie auch in der Forschungsliteratur als eine ,Rückkehrbewegung in die Stadt gehandelt. Dies gilt sowohl für die frühen Gentrifizierungsprojekte, wie 
zum Beispiel das Viertel Society Hill in Philadelphia, die mit erheblicher staatlicher Förderung nach der Gesetzgebung zur Stadterneuerung durchgeführt wurden, als auch für spätere Projekte, wie etwa Federal Hill in Baltimore oder Capitol Hill in Washington, die primär privatwirtschaftliche Phänomene der 1970er waren. All diese Beispiele wurden zu Symbolen für eine angebliche Wanderung der Mittel- und Oberschicht aus dem suburbanen Raum zurück in die Stadt.[5] Dass die Gentrifizierer desillusionierte Vorstädter seien, bleibt jedoch eine bislang nicht überprüfte, wenn auch weit verbreitete Annahme. Bereits 1966 stellte Herbert Gans fest: „Ich kenne keine Studie, aus der hervorgeht, wie viele Vorstädter tatsächlich durch Stadterneuerungsprojekte zurückgeholt wurden.“ (1968: 287) Obwohl Gans diese Erklärung vor dem Ribicoff-Komitee[6] zur Krise der Städte abgab, scheinen die von ihm angemeldeten Zweifel auf taube Ohren gestoßen zu sein. Studien dieser Art wurden erst ab den späten 1970er Jahren durchgeführt. Dieser Artikel stellt Daten zu Society Hill und anderen wiederbelebten Stadtvierteln vor, prüft die Bedeutsamkeit dieser Ergebnisse mit Blick auf die Theorie der Konsumentensouveränität und zielt darauf ab, unser theoretisches Verständnis der Ursachen von Gentrifizierung zu vertiefen.

\section{Eine Rückkehr aus suburbia?}

Society Hill, einst Schauplatz von William Penns , heiligem Experiment', war bis weit in das 19. Jahrhundert die Wohngegend von Philadelphias Oberschicht. Mit der Industrialisierung und dem Wachstum der Stadt verlor die Lage jedoch an Popularität und die Oberschicht zog zusammen mit der aufsteigenden Mittelschicht Richtung Westen in das Gebiet um den Rittenhouse Square beziehungsweise in die neuen Vororte im Nordwesten und jenseits des Schuylkill River. Society Hill verkam zusehends zum Slum und verblieb in diesem Zustand, bis 1959 ein Sanierungsplan umgesetzt wurde.

Binnen zehn Jahren wurde Society Hill umgestaltet und auf der „historischsten Quadratmeile der Nation“ (so eine Annonce zum 200-jährigen Bestehen der USA) wohnte wieder die Mittel- und Oberschicht der Stadt. Kaum eines der authentisch restaurierten Häuser wechselt nun für weniger als 125.00o Dollar den Besitzer. Angesichts des Enthusiasmus, mit dem die Sanierung vonstattenging, bemerkte der Schriftsteller Nathaniel Burt, die Renovierung alter Häuser sei schließlich eine von Alt-Philadelphias „beliebtesten Drinnensportarten“ - und mit dem Umbau gleichzeitig bewusst der zivilgesellschaftlichen Wiederbelebung dienen zu können, sei der Oberschicht „zu Kopf gestiegen wie Champagner“ (1963: 556f.). Als dieser „Drinnensport“ Schule machte, verbreitete sich in Philadelphia die Legende, in Society Hill kehre die Oberschicht in die Innenstadt zurück (Wolf 1975: 325). Oder wie Burt es so eloquent formuliert hat:

„Die Renaissance von Society Hill [...] ist nur ein Stückchen in einem gigantischen Puzzle, das Philadelphia aus seinem Dornröschenschlaf gerissen hat und verspricht, die Stadt vollständig zu verwandeln. Diese Bewegung, ein wichtiger Teil derer die Rückkehr nach Society Hill ist, wird allgemein als die Renaissance Philadelphias bezeichnet.“ (1963: 539) 


\begin{tabular}{|c|c|c|c|c|c|c|}
\hline Jahr & $\begin{array}{c}\text { Selbe } \\
\text { Adresse }\end{array}$ & $\begin{array}{c}\text { Anderes } \\
\text { Stadtviertel }\end{array}$ & Vorort & $\begin{array}{c}\text { Außerhalb des } \\
\text { Ballungsgebiets }\end{array}$ & $\begin{array}{c}\text { Nicht } \\
\text { zuzuordnen }\end{array}$ & Gesamt \\
\hline 1964 & 5 & 9 & 0 & 0 & 0 & 14 \\
\hline 1965 & 3 & 17 & 7 & 0 & 0 & 27 \\
\hline 1966 & 1 & 25 & 4 & 0 & 2 & 32 \\
\hline 1969 & 1 & 9 & 2 & 0 & 0 & 12 \\
\hline 1972 & 1 & 12 & 1 & 2 & 0 & 16 \\
\hline 1975 & 0 & 1 & 0 & 0 & 0 & 1 \\
\hline Gesamtzahl & 11 & 73 & 14 & 2 & 2 & 102 \\
\hline Prozentualer Anteil & 11 & 72 & 14 & 2 & 2 & 100 \\
\hline
\end{tabular}

Noch im Juni 1962 stammte weniger als ein Drittel der Familien, die Eigentum zum Zweck der Sanierung erwarben, aus dem suburbanen Raum[7] (Greenfield \& Co. 1964: 192). Aber seit 1960 die ersten Bewohner begonnen hatten, Häuser zu sanieren, wurde allgemein erwartet, dass der Anteil der Vorstädter steil ansteigen werde, wenn die Gegend erst einmal bekannter und eine Adresse in Society Hill ein begehrtes Gut geworden sei. Nach 1962 wurden jedoch keine amtlichen Daten mehr erhoben. Die Daten in der nachfolgenden Tabelle sind anhand der Vorgangsakten der Redevelopment Authority of Philadelphia (der zuständigen Behörde, Anm. d. Übers.) zusammengestellt. Sie decken den Zeitraum bis $1975 \mathrm{ab}$ (das Jahr, in dem das Projekt im Wesentlichen abgeschlossen war) und umfassen ein Sample von 17 Prozent der sanierten Wohngebäude (Tab. 1).

Diesen Ergebnissen zufolge scheinen die Gentrifizierer tatsächlich nur zu einem kleinen Teil aus suburbia zu stammen: im Fall von Society Hill 14 Prozent, verglichen mit 72 Prozent, die aus einer anderen Gegend innerhalb der Stadtgrenzen zugezogen sind. Eine statistische Aufschlüsselung dieser letztgenannten Gruppe zeigt, dass 37 Prozent bereits vorher in Society Hill wohnten und 19 Prozent aus dem Quartier rund um den Rittenhouse Square kamen. Der Rest stammte aus verschiedenen, während des letzten Jahrhunderts eingemeindeten Mittel- und Oberschichtvororten (z. B. Chestnut Hill, Mt. Airy, Spruce Hill). Dies deutet auf eine Konsolidierung der Wohnlagen der weißen Ober- und Mittelschicht hin und nicht auf eine Rückkehr aus den heutigen suburbanen Räumen.[8] Zahlen aus Baltimore und Washington D.C. zum Prozentsatz der in die Stadt zurückziehenden Vorstädter stützen die Daten aus Society Hill (Tab. 2).

Es mag sich in Philadelphia und anderswo tatsächlich eine urbane Renaissance vollziehen, aber um eine signifikante Rückkehrbewegung aus dem suburbanen Raum im eigentlichen Sinn handelt es sich dabei nicht. Dies

\begin{tabular}{|l|c|c|}
\hline Stadt & $\begin{array}{c}\text { Prozent } \\
\text { Stadtbewohner }\end{array}$ & $\begin{array}{c}\text { Prozent } \\
\text { Vorstädter }\end{array}$ \\
\hline $\begin{array}{l}\text { Philadelphia } \\
\text { Society Hill }\end{array}$ & 72 & 14 \\
\hline $\begin{array}{l}\text { Baltimore } \\
\text { Homestead Properties }\end{array}$ & 65,2 & 27 \\
\hline $\begin{array}{l}\text { Washington D.C. } \\
\text { Mount Pleasant }\end{array}$ & 67 & 18 \\
\hline $\begin{array}{l}\text { Washington D.C. } \\
\text { Capitol Hill }\end{array}$ & 72 & 15 \\
\hline
\end{tabular}

Tab. 1 Vorheriger Wohnort der Gentrifizierer in Society Hill, 1964-1975
Tab. 2 Herkunft der Gentrifizierer in drei Städten (Quelle: Baltimore City Department of Housing and Community Development (1977), Gale (1976, 1977)) 
wiederlegt zwar nicht die Hypothese, Gentrifizierung sei das Ergebnis von Konsumentensouveränität, aber sie bedarf wohl einiger Einschränkungen und Verfeinerungen. Natürlich ist es möglich - und sogar wahrscheinlich -, dass sich jüngere Menschen, die zu Ausbildungszwecken in die Stadt gezogen sind, gegen eine Rückkehr nach suburbia entscheiden. Wenn dies als maßgebliche Erklärung dienen soll, ergibt sich allerdings das Problem, dass Gentrifizierung nicht allein ein nordamerikanisches Phänomen, sondern auch in zahlreichen Städten überall in Europa zu beobachten ist (siehe z. B. Pitt 1977), wo die mittelschichtsgeprägte Suburbanisierung ein wesentlich geringeres Ausmaß hatte und die Beziehung zwischen Umland und Innenstadt eine gänzlich andere ist.[9] Nur die von Ley (1978) aufgestellte, eher allgemeingesellschaftliche Hypothese zu postindustriellen Städten ist breit genug angelegt, um den Prozess auch international zu erfassen, stellt aber eine Position dar, die recht drastische Implikationen nach sich zieht. Wenn sich Gentrifizierung tatsächlich mit kulturell bedingten Entscheidungen und Präferenzen der Konsumenten erklären lässt, liefe dies entweder auf die Hypothese hinaus, dass sich individuelle Präferenzen nicht allein auf nationaler Ebene, sondern auch international im Gleichklang ändern - ein düsteres Bild der menschlichen Natur und kulturellen Besonderheit - oder, dass es übergeordnete Zwänge gibt, die stark genug sind, die in den Präferenzen der Konsumenten angelegte Individualität zu überformen. Falls Letzteres zuträfe, wäre das Konzept der Konsumentenpräferenz bestenfalls widersprüchlich: Ein Prozess, der zuerst im Sinne individueller Konsumvorlieben verstanden wurde, soll nun als das Ergebnis kultureller Eindimensionalität erklärt werden. Die theoretische Tragfähigkeit des Konzepts kann nur gerettet werden, wenn wir es nicht auf individuelle, sondern auf kollektive gesellschaftliche Präferenzen beziehen.

Diese Wiederlegung der neoklassischen Sicht auf Gentrifizierung stellen lediglich eine zusammenfassende Kritik dar und sind bei weitem nicht vollständig. Allerdings legen sie uns nahe, ein weiter gefasstes Verständnis von Gentrifizierung zu entwickeln, denn der Gentrifizierer ist als Konsument nur einer von vielen am Prozess beteiligten Akteuren. Gentrifizierung allein über das Handeln der Gentrifizierer zu erklären und dabei die Rolle der Developer und Bauträger, Kreditgeber, Behörden, Immobilienmakler, Vermieter und Mieter außer Acht zu lassen, ist ein unverhältnismäßig enger Ansatz. Eine breiter angelegte Theorie der Gentrifizierung muss die Rolle der Produzenten wie auch die der Konsumenten berücksichtigen, und wenn man dies tut, dann zeigt sich, dass die Bedürfnisse der Produktionsseite - insbesondere das, Profit zu machen - Gentrifizierung entscheidender vorantreiben als die Präferenzen der Konsumenten. Damit soll nicht in irgendeiner naiven Weise behauptet werden, Konsum sei die automatische Folge von Produktion oder Konsumentenpräferenzen seien ein völlig passiver, durch Produktion generierter Effekt. Dies liefe auf eine Theorie der Produzentensouveränität hinaus, die kaum weniger einseitig wäre als ihr neoklassisches Gegenstück. Die Beziehung zwischen Produktion und Konsum ist vielmehr symbiotischer Natur, aber es ist eine Symbiose, die von der Produktionsseite dominiert wird. Die Präferenzen der Konsumenten und die Nachfrage nach gentrifiziertem Wohnraum lassen sich schließlich auch erzeugen - und genau das ist in Society Hill geschehen.[10] Obwohl sie für das Ingangsetzen des konkreten 
Prozesses und somit auch für die Erklärung, warum Gentrifizierung überhaupt stattfindet, zweitrangig sind, tragen die Präferenzen und die Nachfrage der Konsumenten entscheidend zum letztendlichen Erscheinungsbild und Charakter sanierter Viertel bei, das heißt zum Unterschied zwischen Society Hill und, sagen wir, dem New Yorker Stadtteil SoHo.

Die sogenannte urbane Renaissance wurde eher von ökonomischen als von kulturellen Kräften angeschoben. Bei der Entscheidung, ein innerstädtisches Gebäude zu sanieren, steht eine Konsumentenpräferenz klar im Vordergrund - der Wunsch nach Profit oder, präziser gesagt, nach einer guten Geldanlage. Egal ob Gentrifizierer diesen Wunsch so äußern oder nicht, er ist hier zentral, denn bei absehbarem finanziellem Verlust würde kaum jemand eine Sanierung überhaupt in Erwägung ziehen. Eine Theorie der Gentrifizierung muss daher plausibel erklären, warum Sanierung in einigen Quartieren rentabel ist und in anderen nicht. Welche Bedingungen müssen für Rentabilität gegeben sein? Auf Konsumentensouveränität beruhende Erklärungsansätze gingen stillschweigend davon aus, dass es Gegenden gibt, die ,gentrifizierungsreif sind, wo doch genau dies der Erklärung bedurfte.

Bevor wir uns einer detaillierteren Beschreibung des Prozesses zuwenden, ist es hilfreich, einen Schritt zurück zu gehen und Gentrifizierung im größeren historischen und strukturellen Kontext von Kapitalinvestition und Stadtentwicklung zu beleuchten. $\mathrm{Zu}$ untersuchen sind dabei vor allem die generellen Merkmale von Investitionen in die gebaute Umwelt.

\section{Investitionen in die gebaute Umwelt}

In einer kapitalistischen Ökonomie werden Grund und Boden sowie darauf errichtete Bauten zu Waren. Als solche weisen sie gewisse Eigentümlichkeiten auf, wovon drei für unsere Diskussion besonders bedeutsam sind. Erstens verleihen die privaten Eigentumsrechte dem Besitzer eine quasi monopolistische Verfügungsgewalt über das Land und die Bauten darauf, also darüber, welcher Nutzung eine bestimmte Fläche zugeführt wird.[11] Von dieser Bedingung lässt sich die Funktion der Grundrente ableiten. Zweitens sind Grund und Boden wie auch Bauten zu seiner Nutzung zwar räumlich, aber keinesfalls in ihrem Wert fixiert. Der Wert von gebauten Strukturen unterliegt all den üblichen Einflüssen - aber es gibt einen entscheidenden Unterschied. Einerseits wirkt sich der Wert der auf einem Grundstück sowie auf den umliegenden Flächen errichteten Strukturen auf die Grundrente aus, die Eigentümer erzielen können. Andererseits, da Grund und Boden und die Bauten darauf untrennbar verbunden sind, spiegelt der Preis, zu dem Gebäude den Besitzer wechseln, die Höhe der Grundrente. Anders als die darauf gebauten Strukturen benötigt das Grundstück selbst „keine Wartung, um weiterhin Nutzungspotenzial zu haben“ (Harvey 1973: 158-159) und behält daher seinen potenziellen Wert. Drittens ist zwar der Grund und Boden von dauerhaftem Bestand, die darauf gebauten Strukturen sind es jedoch nicht. Allerdings erstrecken sich in der Regel sowohl die physische Haltbarkeit als auch die ökonomische Lebensdauer über sehr lange Zeiträume. Es dauert mindestens 25 Jahre, meist wesentlich länger, bis ein Gebäude dem physischen Verfall anheimfällt, und ebenso lange mag es dauern, bis es sich in ökonomischer (nicht buchhalterischer) Hinsicht gerechnet 
hat. Daraus lassen sich mehrere Schlüsse ziehen: In einer entwickelten kapitalistischen Ökonomie erfordern Investitionen in die gebaute Umwelt hohe anfängliche Aufwendungen. Deshalb ist davon auszugehen, dass Geldinstituten eine wichtige Rolle auf dem städtischen Grundstücksmarkt zukommt (Harvey 1973: 159) und die Spielarten der Kapitalentwertung eine wichtige Variable darstellen, um zu ermitteln, ob und in welchem Umfang der Verkaufspreis eines Gebäudes das Grundrentenniveau widerspiegelt. Diese Aspekte werden im nächsten Abschnitt von zentraler Bedeutung sein.

In einer kapitalistischen Ökonomie ist Profit der Erfolgsmaßstab und Wettbewerb der Mechanismus, der Erfolg oder Misserfolg in Wachstum oder Niedergang übersetzt. Alle Einzelunternehmen müssen nach immer höheren Profiten streben, um durch profitable Betätigung die Akkumulation immer größerer Kapitalmengen zu ermöglichen. Andernfalls können sie sich keine fortschrittlicheren Produktionsverfahren leisten und geraten so gegenüber ihren Mitbewerbern ins Hintertreffen. Dies führt letztendlich entweder in den Bankrott oder zum Zusammenschluss zu einem größeren Unternehmen. Dieses Streben nach Profitsteigerung setzt sich auf der gesamtwirtschaftlichen Ebene in langfristiges Wachstum um. Allgemeine ökonomische Stabilität ist daher gleichbedeutend mit gesamtwirtschaftlichem Wachstum. Vor allem dann, wenn dem Wirtschaftswachstum anderswo im Industriesektor Grenzen gesetzt sind, wird die gebaute Umwelt vielfach zum profitablen Investitionsobjekt, was an der Suburbanisierungsgeschichte dieses Jahrhunderts besonders deutlich wird. In ihrem Falle bestand die Antwort auf den fortwährenden Bedarf an Kapitalakkumulation nicht in Wachstum an Ort und Stelle, sondern in der räumlichen Ausdehnung. Aber Suburbanisierung zeigt auch sehr anschaulich die Zweiseitigkeit von Investitionen in die gebaute Umwelt, denn ebenso, wie sie als Mittel zur Kapitalakkumulation dienen, können sie weitere Akkumulation auch behindern. Zum Hemmnis werden sie kraft der oben beschriebenen Merkmale, das heißt aufgrund der quasi-monopolistischen Verfügungsgewalt über den Grund und Boden, der Gebundenheit der Investitionen und des langen Amortisationszeitraums: Die quasi-monopolistische Verfügungsgewalt des Eigentümers über sein Grundstück kann den Verkauf des Geländes als Bauland verhindern. Die gebundenen Investitionen führen dazu, dass neue Bauprojekte auf andere, oftmals weniger vorteilhafte Standorte ausweichen müssen, und sie verhindern eine Sanierung, bevor das investierte Kapital das Ende seines ökonomischen Lebenszyklus erreicht hat. Und die lange Amortisationsdauer des in die gebaute Umwelt investierten Kapitals kann als Investitionshindernis wirken, so lange andere Wirtschaftssektoren mit kürzeren Amortisationszeiträumen profitabel bleiben. Gegen Ende des 19. Jahrhunderts wurde die frühindustrielle Stadt zu solch einem Investitionshindernis, was letztlich dazu führte, dass eher das städtische Umland erschlossen wurde, statt die bestehenden innerstädtischen Standorte weiterzuentwickeln.

In den meisten Städten des Ostens der USA wies die Bodenwertkurve während des 19. Jahrhunderts die klassische Kegelform auf, mit dem höchsten Punkt im Stadtzentrum und einem zur Peripherie hin abfallenden Verlauf. Dieses Muster konnte Hoyt (1933) in Chicago feststellen. Mit fortschreitender Stadtentwicklung verschiebt sich die Bodenwertkurve nach außen und 
oben: Grundstücke im Stadtzentrum gewinnen an Wert, während die Basis des Kegels breiter wird. Bodenwerte entwickeln sich tendenziell analog zu den langen Zyklen der Ökonomie, das heißt sie steigen am schnellsten in Phasen besonders starker Kapitalakkumulation und fallen bei rückläufiger Konjunktur vorübergehend ab. Da die Suburbanisierung erheblicher Kapitalinvestitionen in Grund und Boden, Bautätigkeit, Verkehrsanbindung und so weiter bedurfte, folgte sie in der Tendenz ebenfalls diesem zyklischen Trend. Angesichts der Notwendigkeit, den Umfang ihrer Produktionstätigkeit auszuweiten, verlagerten sich Industriebetriebe, die am bisherigen Standort aus verschiedenen Gründen nicht weiter expandieren konnten oder wollten, aus der Innenstadt an die Basis des Bodenwertkegels, wo eine großflächige räumliche Erweiterung sowohl machbar als auch verhältnismäßig preisgünstig war. Die Alternative - eine grundlegende Sanierung und Modernisierung des bereits bebauten Areals - wäre für das Privatkapital ein zu kostspieliges Unterfangen gewesen und so floss das Industriekapital zunehmend in die Vororte. Dieser Abfluss von Industriekapital setzte nach der schweren Wirtschaftskrise von 1893 bis 1897 ein, gefolgt von einer beträchtlichen Abwanderung von Kapital für den Wohnungsbau. Die einzige signifikante Ausnahme von dieser Abwanderung von Baukapital waren die zentralen Geschäftsviertel der bereits etablierten Städte, wo in den 1920er Jahren in großem Stil Bürohochhäuser errichtet wurden. Wie im Folgenden gezeigt wird, wirkte sich dieser Kapitalabfluss in Richtung der mehr Ertrag versprechenden Vororte nachteilig auf die Innenstadt aus. Eine Kombination aus Vernachlässigung und einem aufgrund hoher Risiken und geringer Renditen konzertiert betriebenen Abzug von Kapital seitens der Investoren läutete eine lange Periode des Verfalls und des Ausbleibens neuer Kapitalinvestitionen in die Innenstadt ein.

Die Bodenwerte in der Innenstadt fielen im Verhältnis zum zentralen Geschäftsviertel und den Vororten. Gegen Ende der 1920er Jahre konnte Hoyt für Chicago ein neu entstandenes „Tal in der Bodenwertkurve zwischen The Loop [12] und den äußeren Wohngebieten“ identifizieren (siehe Abb. 1). Dieses Tal „markiert die Lage der Gebiete, wo die Gebäude meist über vierzig Jahre alt sind und die Bewohner hinsichtlich ihrer Mietzahlungsfähigkeit ganz unten rangieren" (Hoyt 1933: 356-358). Von den 1940er bis zu den 1960er Jahren, den Jahrzehnten nachhaltigster Suburbanisierung, wurde dieses Tal in der Bodenwertkurve immer breiter und tiefer, da es weiterhin an produktiven Kapitalinvestitionen fehlte. Gegen Ende der 1960er Jahre dürfte dieses Tal in Chicago bis zu sechs Meilen breit gewesen sein (McDonald/Bowman 1979). Befunde aus anderen Städten deuten darauf hin, dass diese Kapitalentwertung und die daraus resultierende Verbreiterung des Bodenwerttals alle älteren Städte des Landes betraf (Davis 1965; Edel/ Sclar 1975) und dort jene Slums und Ghettos entstehen ließ, die in den 1960er Jahren von der schon lange abgewanderten suburbanen Mittelschicht plötzlich als ,Problem‘ ausgemacht wurden.

Eine Theorie der Gentrifizierung wird die historischen Mechanismen der Kapitalentwertung in der Innenstadt im Detail erklären und darlegen müssen, auf welche Weise genau dies profitable Reinvestitionen möglich macht. Der entscheidende Zusammenhang ist hier das Verhältnis zwischen Bodenwert und Immobilienwert. Derzeit scheinen diese beiden Begriffe 
Abb. 1 Entwicklung der Bodenwerte in Chicago (nach Hoyt 1933)

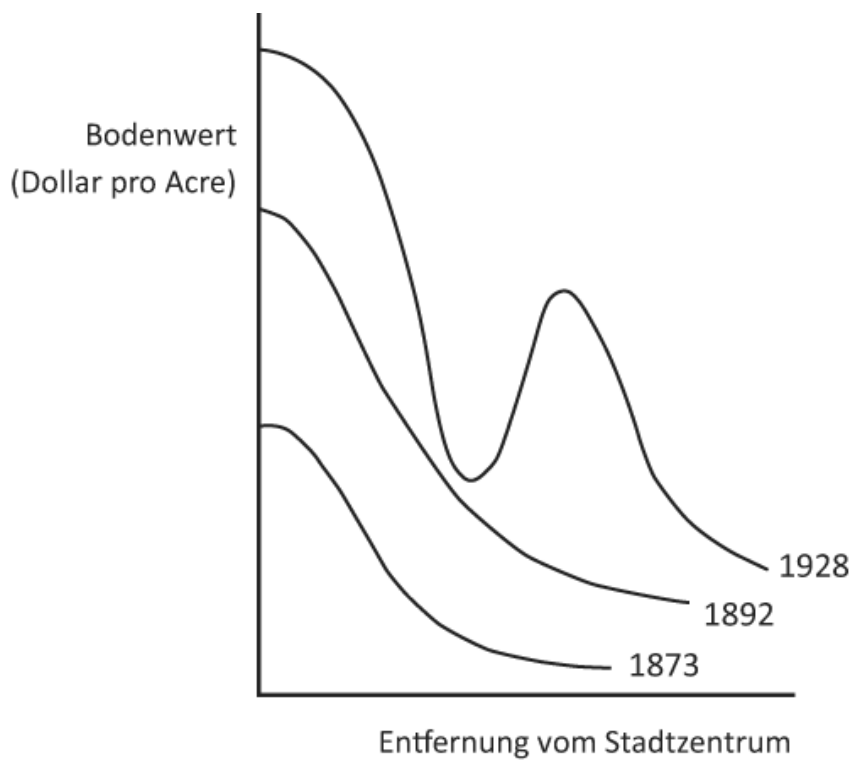

allerdings nicht hinreichend ausgearbeitet. Hoyt verstand unter Bodenwert eine Mischkategorie, bestehend aus dem Preis unbebauter Grundstücke und den erwarteten zukünftigen Einnahmen aus ihrer Nutzung, wobei er die Art der künftigen Nutzung einfach voraussetzte. Der Begriff Immobilienwert hingegen bezeichnet üblicherweise den Preis, zu dem ein Gebäude verkauft wird, den Wert des Grundstücks miteingeschlossen. Um die Beziehung zwischen dem Bodenwert und dem Wert von Gebäuden präziser herauszuarbeiten, ist es daher notwendig, diese beiden Wertmaßstäbe in vier separate, aber miteinander zusammenhängende Kategorien aufzuschlüsseln. Diese vier Kategorien - Gebäudewert, Verkaufspreis, kapitalisierte Grundrente und potenzielle Grundrente - bleiben nämlich gänzlich oder partiell unbestimmt beziehungsweise ununterscheidbar, wenn man allein mit den Dachbegriffen Bodenwert und Immobilienwert operiert.

- Gebäudewert. In Übereinstimmung mit ihrer Betonung der Konsumentenpräferenz deutet die neoklassische Theorie Preise als das Ergebnis von Angebots- und Nachfragebedingungen. Wenn aber, wie oben angedeutet, der Wunsch nach hohen Renditen auf produktives Anlagekapital der primäre Antrieb für Gentrifizierung ist, dann sind die spezifischen Produktionskosten (und nicht nur die Menge des Endproduktes, also das Angebot) der zentrale Faktor für die Preisermittlung. Anders als in der neoklassischen Theorie wird es daher erforderlich sein, zwischen dem Wert eines Hauses und seinem Preis zu trennen. In Anlehnung an die klassische politische Ökonomie (Smith und Ricardo), und in ihrer Folge Marx, geht dieser Beitrag axiomatisch von einer Arbeitswerttheorie aus: Der Wert einer Ware bemisst sich an der für ihre Herstellung erforderlichen gesellschaftlich notwendigen Arbeit. Erst auf dem Markt wird der Wert zum Preis. Und obwohl der Preis eines Hauses seinen Wert widerspiegelt, können die beiden Größen nicht automatisch gleichgesetzt werden, da der Preis auch von Angebots- und Nachfragebedingungen beeinflusst wird. Folglich bestimmen Wertaspekte, das heißt die Menge der gesellschaftlich notwendigen Arbeitskraft, das Niveau, um das herum sich der Preis bewegt. Bei Wohnraum ist die Situation komplexer, weil das einzelne Haus von Zeit zu Zeit wieder auf den Markt kommt, um weiterverkauft zu werden. Der Wert des Hauses wird somit auch davon 
abhängen, in welchem Umfang es durch Abnutzung an Wert verliert beziehungsweise durch Aufwertung an Wert gewinnt. Letzteres ist dann der Fall, wenn zusätzliche Arbeit für Instandhaltung, Erneuerung, Ausbau und so weiter aufgewendet wird.

- Verkaufspreis. Was die Sache bei Wohnraum noch weiter verkompliziert, ist, dass der Verkaufspreis nicht allein den Wert des Hauses an sich abbildet, sondern auch Mieterträge berücksichtigt, da Grundstücke üblicherweise zusammen mit den darauf errichteten Strukturen verkauft werden. Hier wäre es besser, statt von Bodenwert von Grundrente zu sprechen, denn anders als ein echter Warenwert bildet der Grundstückspreis den Umfang der aufgewendeten Arbeitskraft nicht ab.

- Grundrente und kapitalisierte Grundrente. Die Grundrente ist ein Anspruch, den Grundeigentümer gegenüber den Nutzern ihres Grundstücks erheben. Für die Developer impliziert sie die Minderung des Mehrwerts, der auf dem Grundstück über die Errichtungskosten hinaus geschaffen wurde. Die kapitalisierte Grundrente ist die tatsächliche Menge an Grundrente, die sich der Eigentümer bei einer gegebenen Nutzung seines Grundstücks aneignet. Wenn es sich um ein Mietobjekt handelt, mit dem der Vermieter auf seinem Grund und Boden eine Dienstleistung erbringt, vermischen sich die Produktions- und Eigentumsfunktion und die Grundrente wird zu einer noch schwerer greifbaren, aber doch real existierenden Kategorie. Hier erzielt der Vermieter seine kapitalisierte Grundrente größtenteils in Form von Mieteinnahmen. Bei selbstgenutztem Wohneigentum kapitalisiert sich die Grundrente, wenn die Immobilie verkauft wird, und erscheint somit als Teil des Verkaufspreises. Ergo, Verkaufspreis = Gebäudewert + kapitalisierte Grundrente.

- Potenzielle Grundrente. Bei einer bestimmten Nutzung kann aus einem Grundstück beziehungsweise Quartier eine bestimmte Menge an kapitalisierter Grundrente gezogen werden. Aus Standortgründen ließe sich auf solch einer Fläche durch eine andere Art der Nutzung meist eine höhere kapitalisierte Grundrente erzielen. Die potenzielle Grundrente bezeichnet den Betrag, der kapitalisiert werden könnte, wenn die Fläche der ,höchsten und besten Nutzung' zugeführt würde. Dieser Begriff ist von zentraler Bedeutung, wenn man Gentrifizierung erklären will.

Mit den genannten Konzepten lässt sich der historische Prozess umreißen, der bestimmte Viertel ,gentrifizierungsreif' gemacht hat.

\section{Kapitalentwertung in der Innenstadt}

Der physische Verfall und die ökonomische Entwertung innerstädtischer Viertel sind ein absolut logisches, ,rationales ' Resultat dessen, wie der Grundstücks- und Wohnungsmarkt funktioniert. Das soll nicht heißen, dies sei von Natur aus so, ist doch der Markt selbst ein gesellschaftliches Produkt. Der Niedergang von Quartieren ist keineswegs unvermeidbar, sondern

„das Ergebnis identifizierbarer privater und öffentlicher Investitionsentscheidungen. [...] Auch wenn es keinen Napoleon gibt, der die 
Macht hat, über das Schicksal eines Viertels zu bestimmen, verfügen die Investitions- und Entwicklungsakteure der Immobilienbranche über genügend Kontrolle und sind [als Akteursgruppe, Anm. d. Übers.] hinreichend handlungsfähig, um nicht nur zu reagieren, sondern mit ihren Entscheidungen das Marktgeschehen faktisch zu gestalten.“ (Bradford/Rubinowitz 1979: 79)

Was folgt, ist ein recht schematischer Versuch, den historischen Niedergang innerstädtischer Viertel anhand der beteiligten Institutionen, Akteure und ökonomischen Kräfte zu erklären. Hierfür gilt es, einige ins Auge springende Prozesse zu identifizieren, die für die verschiedenen Phasen des Niedergangs charakteristisch sind, ohne jedoch behaupten zu wollen, dass dies in jedem Fall genau so abläuft. Die tagtäglichen Dynamiken des Niedergangs sind komplex und wurden an anderer Stelle sehr detailliert untersucht, insbesondere hinsichtlich der Beziehung zwischen Vermietern und Mietern (Stegman 1972). Was dieses Schema jedoch leisten soll, ist, einen allgemeinen Erklärungsrahmen zu bieten, mit dem sich für jedes Quartier nachvollziehen lässt, was dort konkret passiert. Die Ausgangsvermutung ist, dass die betroffenen Nachbarschaften hinsichtlich Alter und Beschaffenheit der Wohngebäude relativ homogen sind, was auf Quartiere, die Sanierung erfahren, in der Tat zuzutreffen pflegt.

1. Neubau und erster Nutzungszyklus. In einem Neubauquartier spiegelt der Preis einer Immobilie den Wert des Bauwerks und der Erschließungsmaßnahmen plus die höhere Grundrente, die sich der vorherige Grundstücksbesitzer aneignet. Im Verlauf des ersten Nutzungszyklus wird die Grundrentein der Regel ansteigen, während sich dieStadt weiter ausdehnt, und der Gebäudewert wird, wenn überhaupt, nur sehr langsam zu sinken beginnen. Folglich steigt der Verkaufspreis. Aber über kurz oder lang setzt der kontinuierliche Wertverlust des Hauses ein, und zwar aus drei Gründen: Steigerung der Arbeitsproduktivität, stilistische Veralterung und physische Abnutzung. Die gesteigerte Arbeitsproduktivität ist vor allem das Ergebnis technologischer Innovation und einer veränderten Organisation des Arbeitsprozesses. Diese Fortschritte erlauben es, ein vergleichbares Bauwerk zu einem niedrigeren Wert zu errichten als es sonst möglich gewesen wäre. Vorgefertigte Bauten und die industrielle Fertigung von Bauteilen allgemein (statt des Bauens in situ) sind die jüngsten Beispiele hierfür. Stilistische Veralterung als Triebfeder für die kontinuierliche Wertminderung ist auf dem Immobilienmarkt sekundär und kann mitunter auch zu einem Wertzuwachs führen, da viele alte Baustile heute begehrter sind als die neuen. Physische Abnutzung wirkt sich auch auf den Wert von Wohnraum aus. Hier muss allerdings unterschieden werden: Geht es um die regelmäßig durchzuführenden werterhaltenden Schönheitsreparaturen (z. B. Neuanstrich von Türen und Fensterrahmen, Innenrenovierung), um größere Reparaturen, die seltener, aber mit höheren Kosten verbunden sind (z. B. Austausch der elektrischen oder Rohrleitungen) oder um bauliche Instandsetzung, ohne die das Gebäude verfällt (z. B. Erneuerung des Dachs, Austausch schwammbefallener Dielen). In der Wertminderung einer Immobilie nach einem Nutzungszyklus spiegelt sich, dass nicht mehr allein die 
turnusmäßigen Schönheitsreparaturen anstehen, sondern auch eine Reihe größerer Reparaturmaßnahmen, die erhebliche Investitionen erfordern. Diese Wertminderung führt nun dazu, dass der Preis im Verhältnis zu Neubauten sinkt, aber das Ausmaß dieser Preisminderung wird davon abhängen, inwieweit sich in der Zwischenzeit auch die Grundrente verändert hat.

2. Selbstnutzung versus Vermietung. Zweifellos gelingt es den Bewohnern vieler Quartiere, größere Reparaturen durchzuführen und den Wert des Wohnraums in ihrer Gegend zu erhalten oder gar zu steigern. Diese Quartiere bleiben stabil. Ebenso offensichtlich gibt es Eigenheimviertel, die eine erste Wertminderung erfahren. Im Wissen, dass ohne Reparaturen der Verfall einsetzt, werden Hausbesitzer vermutlich eher verkaufen und sich ein neueres Objekt suchen, in dem ihr Geld sicherer angelegt ist. An diesem Punkt, nach einem ersten oder zweiten Nutzungszyklus, besteht die Tendenz, dass sich das Viertel zu einer Mietergegend wandelt, es sei denn, Reparaturmaßnahmen werden durchgeführt. Und da Vermieter Gebäude zu anderen Zwecken nutzen als Eigenheimbewohner, wird sich folglich ein anderes Instandhaltungsmuster ergeben. Auf dem Immobilienmarkt sind Eigenheimbewohner zugleich Konsumenten wie auch Investoren. Als Letztere erzielen sie ihre Rendite primär dadurch, dass der Verkaufspreis über dem ursprünglichen Kaufpreis liegt. Der Vermieter hingegen bezieht seine Rendite vor allem in Form von Miete und mag unter bestimmten Bedingungen einen geringeren Anreiz für Reparaturen haben, so lange sich noch Miete einstreichen lässt. Das soll nicht heißen, dass mangelhafte Instandhaltung ihres Eigentums für Vermieter typisch ist. Neuere Wohnkomplexe und sogar Altbauwohnungen, die stark nachgefragt sind, können sehr gut gepflegt sein. Allerdings ist, worauf Ira Lowry hingewiesen hat, „mangelnde Instandhaltung eine überaus vernünftige Reaktion eines Vermieters auf einen rückläufigen Markt“ (1960: 367). Und da die Umstellung von Selbstnutzung auf Vermietung in der Regel mit einem rückläufigen Markt einhergeht, ist ein gewisses Maß an mangelnder Instandhaltung erwartbar.

Durch mangelhafte Instandhaltung lässt sich ein Kapitalüberschuss erwirtschaften, der anderswo investiert werden kann. Sei es, dass er in andere innerstädtische Immobilien investiert wird, dem Kapital der Developer in die Vororte folgt oder in einen anderen Wirtschaftszweig fließt. Dauert die mangelhafte Instandhaltung in einem Quartier jedoch an, mag es für Vermieter schwierig werden, ihr Eigentum zu verkaufen, vor allem, weil die größeren Kreditinstitute dann eine geringere Bereitschaft zur Kreditfinanzierung an den Tag legen werden. Verkäufe werden seltener und für den Vermieter teurer. Folglich sinkt der Anreiz, in die Gegend zu investieren, auf das Minimum, um den Ertragsfluss auf dem bestehenden Niveau zu halten. Dieses Muster des Niedergangs lässt sich wohl nur dann umkehren, wenn es zu einem Mangel an hochwertigem Wohnraum kommt, sodass Mieten erhöht werden können und sich bessere Instandhaltung lohnt. Anderenfalls dürfte es eher zu einem Nettokapitalabfluss aus dem Viertel kommen, der zunächst nur gering sein wird, da es für die Vermieter immer noch erhebliche 
Abb. 2 Der Wertminderungszyklus innerstädtischer Quartiere

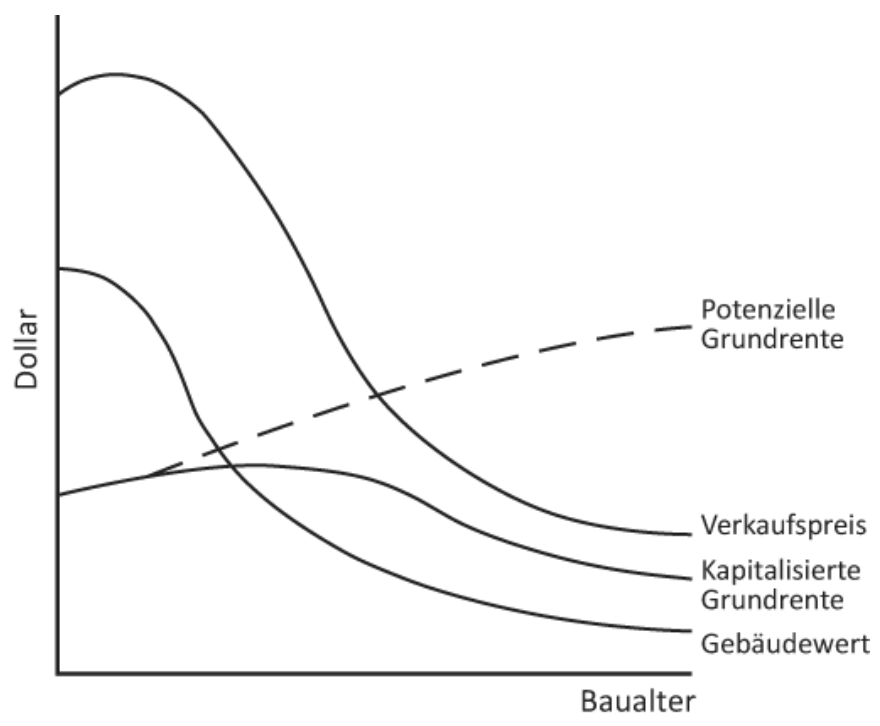

Investitionen zu schützen gilt. Unter diesen Bedingungen wird es für den einzelnen Vermieter beziehungsweise Eigentümer sehr schwer, sich dem Niedergang entgegenzustemmen. Die Gebäudewerte fallen und das Niveau der kapitalisierten Grundrente sinkt in dieser Gegend unter das der potenziellen Grundrente (siehe Abb. 2). Ein Vermieter, der sein Eigentum gut instandgehalten hat, wäre nun gezwungen, mehr als die ortsübliche Durchschnittsmiete zu verlangen, könnte aber kaum darauf hoffen, überdurchschnittlich zahlungskräftige Mieter zu finden, mittels derer sich die volle Grundrente kapitalisieren ließe. Dies ist der berühmte ,Nachbarschaftseffekt ${ }^{\star}$, der sich hier in der Mietenstruktur abbildet.

3. Blockbusting und blow out. Manche Viertel werden der Umwandlung in Mieterquartiere auch entgehen. Dort bleibt die Lage vermutlich relativ stabil oder der Niedergang verläuft sanfter. Im letzteren Fall wäre es selbstgenutztes Wohneigentum, das schlecht instandgehalten wird, allerdings zumeist aufgrund finanzieller Engpässe und nicht als Marktstrategie. Durch sogenanntes blockbusting (das gezielte ,Aufsprengen“ eines Quartiers, Anm. d. Übers.) lässt sich der Niedergang beschleunigen: Hier machen sich Immobilienmakler rassistische Ressentiments zunutze, indem sie in weißen Stadtvierteln, die von sinkenden Verkaufspreisen betroffen sind, Häuser relativ günstig ankaufen und dann mit einem beträchtlichen Aufschlag an schwarze Familien weiterveräußern (welche vielfach den drängenden Wunsch haben, auch einmal ein Eigenheim zu besitzen). Laurenti (1960) zufolge sind die Immobilienpreise in der Regel bereits rückläufig, bevor es zum blockbusting kommt, und beginnen nicht erst zu fallen, weil nun die Hautfarbe der Hauseigentümer eine andere ist. Ist das Viertel jedoch erst einmal ,aufgesprengt', ist ein weiteres Sinken der Gebäudewerte wahrscheinlich, da die zugezogenen Familien infolge der überhöhten Kaufpreise wenig Mittel für Instandhaltung und Hypothekenzahlungen zur Verfügung haben. Blow out ist ein ähnlicher Prozess, der aber ohne die tätige Mitwirkung von Immobilienmaklern auskommt. In ihrer Beschreibung dieses Prozesses, wie er während der 1960er Jahre auf dem Baltimorer Wohnungsmarkt zu beobachten war, verweisen Harvey et al. (1972; siehe auch Harvey 1973: 173) auf die Ausbreitung innerstädtischer Slums - die Verbreiterung des Bodenwerttals -, wodurch 
die an ihrem Rand angrenzenden, bislang intakten Quartiere zwischen dem Slum und den weiter außerhalb liegenden, sicheren Wohnenklaven der oberen Mittelschicht eingekeilt wurden. So eingekeilt, werden die selbstnutzenden Bewohner eines ganzen Viertels sehr wahrscheinlich ihre Eigenheime verkaufen und in den suburbanen Raum fliehen. Die Käufer sind häufig keine Selbstnutzer, sondern wollen vermieten.

4. Redlining. Der mangelnden Instandhaltung folgt ein aktiverer Investitionsabbau, da das Kapital weiter an Wert und das Objekt für den Vermieter an ökonomischer Relevanz verliert. Gebäudewert und kapitalisierte Grundrente fallen, was wiederum die Verkaufspreise noch weiter sinken lässt. Begleitet wird die Desinvestition seitens der Eigentümer von einer ebenso ,rationalen' Desinvestition seitens der Finanzinstitute, die keine Hypothekendarlehen mehr in das Viertel vergeben. Größere Geldinstitute, die Darlehen mit geringerem Eigenkapitalanteil und zu niedrigeren Zinsen anbieten, stellen fest, dass sich im suburbanen Raum, wo die Wahrscheinlichkeit einer Zwangsversteigerung kleiner und das Risiko sinkender Immobilienwerte geringer ist, höhere Renditen erzielen lassen. An die Stelle der großen Finanzinstitute treten in der Innenstadt zunächst kleinere, oft örtliche Institute, die auf Risikofinanzierung spezialisiert sind. Wenn ein Quartier von den größeren Instituten ,mit einer roten Linie‘[13] markiert, das heißt auf eine Art schwarze Liste gesetzt wurde, können auch von der Federal Housing Administration (FHA)[14] abgesicherte Kredite dort hinfließen. Obwohl sie Niedergang eigentlich verhindern sollen, haben FHA-Kredite sich oft als ineffektiv erwiesen und trugen manchenorts sogar zum Niedergang bei (Bradford/ Rubinowitz 1975: 82). Die Kredite ermöglichen, dass Immobilien den Besitzer wechseln, aber bieten kaum Anreize, in Instandhaltung zu investieren, und so geht der Niedergang einfach nur reibungsloser vonstatten. Schließlich weigern sich auch mittelgroße und Kleininvestoren sowie Hypothekenversicherungen, das Viertel weiter zu bedienen.

Vandalismus beschleunigt den Wertverfall noch weiter und wird vor allem dann zum Problem, wenn Immobilien zwischen Mietverhältnissen zeitweise leer stehen (Stegman 1972: 60). Aber auch bewohnte Gebäude können davon betroffen sein, insbesondere, wenn sie schlecht instandgehalten oder systematisch , ausgepresst ' werden. In diesem Stadium ist es gängige Praxis, Gebäude neu aufzuteilen, um mehr Mieteinheiten zu schaffen. Dadurch, so hofft der Vermieter, lässt sich das Gebäude während der noch verbleibenden Jahre intensiver (und rentabler) nutzen. Aber irgendwann wird er seine Investitionen vollständig zurückfahren, Reparaturen verweigern und nur noch die Ausgaben tätigen - und das oft auch nur sporadisch -, die er tätigen muss, damit das Haus weiterhin Miete abwirft.

5. Leerstand. Wenn Vermieter nicht mehr genug Miete einstreichen können, um die notwendigen Ausgaben (Betriebskosten und Steuern) zu decken, werden Häuser dem Leerstand und Verfall überlassen. Dieses Phänomen betrifft das gesamte Quartier, nicht nur einzelne Objekte in einer ansonsten stabilen Umgebung. Viele aufgegebene Häuser sind baulich durchaus intakt und dies erscheint paradox. Gebäude werden jedoch nicht leer stehen gelassen, weil sie nicht mehr nutzbar sind, sondern, weil sie nicht 
profitabel genutzt werden können. Die letztendliche Aufgabe des Hauses kann ganz unterschiedliche Auslöser (aber nicht Ursachen) haben, die strikte Durchsetzung der Bauordnung seitens der städtischen Bauaufsicht miteingeschlossen. In diesem Stadium des Verfalls besteht für Vermieter auch ein gewisser Anreiz, ihr Hauseigentum durch Brandstiftung zu zerstören und die beträchtliche Versicherungssumme zu kassieren.

\section{Gentrifizierung: Die Ertragslücke}

Der im obigen Abschnitt zusammenfassend dargelegte Prozess wird gemeinhin, aber irreführenderweise als ,Sickereffekt' (filtering) bezeichnet. Dabei handelt es sich um einen auf dem Wohnungsmarkt verbreiteten Vorgang, der in vielen Quartieren zu beobachten, aber keineswegs universell ist.[15] Hier miteinbezogen wurde dieser Prozess, gerade weil er fast immer der Gentrifizierung vorausgeht, auch wenn er dafür nicht in all seinen Facetten auftreten muss. Ebenso wenig sollte der beschriebene Niedergang als unausweichlich angesehen werden. Wie Lowry ganz richtig betont, ist der Sickereffekt nicht einfach dem „unerbittlichen Lauf der Zeit“ geschuldet, sondern Ergebnis „menschlichen Handelns“ (1960: 370). Im vorigen Abschnitt wurden einige dieser Akteure benannt, ebenso die Marktkräfte, auf die sie reagieren und die sie gleichzeitig selbst mit hervorbringen. Zudem wurde gezeigt, dass der dem Sickereffekt zugrunde liegende objektive Mechanismus in der Entwertung und im Preisverfall des in innerstädtische Wohngebiete investierten Kapitals besteht. Dieser Wertverlust erzeugt die objektiven ökonomischen Bedingungen, unter denen Wiederaufwertung des Kapitals (Gentrifizierung) zu einer ,rationalen' Reaktion des Marktes wird. Von elementarer Bedeutung ist hier das, was ich als Ertragslücke bezeichne.

Die Ertragslücke ist die Kluft zwischen der potenziellen Grundrente und dem Betrag, der sich aus der bestehenden Nutzung des Grundstücks tatsächlich kapitalisieren lässt (siehe Abb. 2). Beim Sickereffekt entsteht die Ertragslücke vor allem durch Kapitalentwertung, wodurch sich der Anteil der kapitalisierbaren Grundrente verringert, sowie durch die fortschreitende Stadtentwicklung und -erweiterung, die historisch zum Anstieg der in Innenstadtlagen potenziell erzielbaren Grundrente geführt haben. Das Tal, das Hoyt 1928 bei seiner Ermittlung der Bodenwerte nachgewiesen hat, lässt sich nun zu einem guten Teil als eben diese Ertragslücke erklären. Nur wenn diese Lücke entsteht, ist auch mit Umbau zu rechnen, denn wenn sich die Grundrente durch die bestehende Nutzung vollständig oder weitestgehend kapitalisieren ließe, brächte Umbau kaum ökonomische Vorteile. Während Sickereffekt und Niedergang des Viertels voranschreiten, vergrößert sich auch die Ertragslücke. Gentrifizierung setzt dann ein, wenn die Lücke so groß geworden ist, dass Developer Häuser billig kaufen, die Baukosten für Sanierungsmaßnahmen sowie Hypotheken- und Baukreditzinsen tragen und das Endprodukt für einen Preis weiterverkaufen können, der ihnen eine zufriedenstellende Rendite einbringt. Nun lässt sich die gesamte Grundrente - oder ein großer Teil davon - kapitalisieren. Das Quartier wurde ,recycelt ${ }^{\star}$ und ein neuer Nutzungszyklus beginnt.

Sobald die Ertragslücke groß genug ist, kommen verschiedene Akteure auf dem Grundstücks- und Immobilienmarkt infrage, um den 
Gentrifizierungsprozess in dem betreffenden Viertel in Gang zu setzen. Und hier kommen wir wieder auf das Verhältnis zwischen Produktion und Konsum zurück, denn es gibt überzeugende empirische Belege dafür, dass der Prozess nicht dadurch ausgelöst wird, dass individuelle Konsumenten ihre von neoklassischen Ökonomen so hoch geschätzten Präferenzen ausleben, sondern durch irgendeine Form des kollektiven sozialen Handelns[16] auf Quartiersebene. Beispielsweise wurden die meisten, wenn nicht gar alle frühen Programme staatlich initiiert, und obwohl der Staat heute eine geringere Rolle spielt, ist er noch immer ein wichtiger Akteur. Heute, in Zeiten der privatwirtschaftlich getragenen Gentrifizierung, ist es eher so, dass ein oder mehrere Finanzinstitute ein jahrelang ,rot markiertes ${ }^{6}$ Viertel von der Negativliste streichen und als potenziellen Markt für Bau- und Hypothekenkredite ins Auge fassen. Alle Konsumentenpräferenzen der Welt bewirken nichts, sofern diese lang ausgetrocknete Geldquelle nicht wieder zu sprudeln beginnt. Hypothekenkapital ist eine Grundvoraussetzung.

Natürlich muss dieses Hypothekenkapital auch von willigen Konsumenten, die die eine oder andere Präferenz zum Ausdruck bringen, als Kredit aufgenommen werden. Voraussetzung sind diese Präferenzen aber nicht, da sie, wie oben gezeigt, gesellschaftlich erzeugt werden können. Zusammen mit den Finanzinstituten agieren professionelle Developer als die kollektiven Initiatoren, die Gentrifizierung in Gang setzen. Ein Developer erwirbt einen beträchtlichen Teil der Immobilien in einem Quartier, saniert sie und verkauft sie dann gewinnbringend weiter. Die einzige signifikante Ausnahme zu dieser Dominanz kollektiven Handelns sind Viertel, die an bereits gentrifizierte Gegenden angrenzen. Dort kann der initiale Impuls zur Sanierung in der Tat von einzelnen Gentrifizierern ausgehen. Ihre Entscheidung, ebenfalls zu sanieren, ist durch den Erfolg im Nachbarquartier motiviert, was allerdings voraussetzt, dass sie tatsächlich an erster Stelle eine lohnende Geldinvestition im Sinn haben. Und auch sie benötigen erst einmal Hypothekenkapital von willigen Geldgebern.

Die bei der Wiederverwertung von Quartieren üblicherweise in Erscheinung tretenden Developer lassen sich in drei Kategorien einteilen: (a) professionelle Entwickler, die Immobilien ankaufen, sanieren und mit Gewinn weiterverkaufen, (b) Selbstnutzer, die Wohneigentum erwerben und sanieren, um es anschließend auch zu bewohnen und (c) Eigentümer, die ihre Immobilie nach der Sanierung vermieten wollen.[17] Professionelle Developer erzielen ihre Kapitalrendite aus dem Weiterverkaufspreis, Vermieter über die Mieteinnahmen. Die Rendite aus dem Verkauf beinhaltet zwei getrennte Formen des Gewinns: Die Kapitalisierung einer höheren Grundrente und den Ertrag aus der Investition von Produktivkapital (nicht zu verwechseln mit dem Profit des Bauunternehmens) (siehe Smith 1979). Professionelle Projektentwickler und sanierende Vermieter spielen eine wichtige Rolle im Gegensatz zur öffentlichen Wahrnehmung waren sie in Society Hill mit Abstand in der Überzahl -, aber wenn es um Sanierung geht, sind selbstnutzende Eigentümer aktiver engagiert als in allen anderen Bereichen des Wohnungsbaus. Dies liegt womöglich primär im Wesen der Gentrifizierung selbst begründet sowie in den oben diskutierten Charakteristika von Investitionen in die gebaute Umwelt. Wie Sanierung findet auch Umbau dort statt, wo sich eine Ertragslücke aufgetan hat, aber im Fall von Umbau sind die maroden 
Bestände entweder zu baufällig oder die noch stehenden Gebäude lassen sich keiner neuen Nutzung zuführen. Während sich die technischen und räumlichen Anforderungen an Industrie- und Gewerbebauten über die letzten 100 Jahre hinweg grundlegend geändert haben, gilt dies für Wohnbauten nicht und baulich intakte Stadthäuser sind, sofern die ökonomischen Bedingungen stimmen, durchaus noch nutzbar. Da aber die Grundstücke bereits bebaut sind und ein komplexes Geflecht an Eigentumsrechten besteht, ist es für professionelle Developer schwer, hinreichend viele Grundstücke und Immobilien zusammenzutragen, damit sich das Engagement lohnt. Sogar Eigentümer, die weitervermieten wollen, neigen dazu, mehrere Objekte gleichzeitig oder nacheinander zu sanieren. Durch die fragmentierte Struktur von Immobilienbesitz wurde der selbstnutzende Eigentümer, so unzulänglich er als Akteur in der Baubranche meist auch agieren mag, zum probaten Vehikel, um entwertete Quartiere der Wiederverwertung zuzuführen.

So gesehen ist Gentrifizierung kein Zufallsereignis oder die unerklärliche Umkehrung irgendeines unausweichlichen Sickereffekts. Im Gegenteil, sie ist erwartbar. Zusammen mit dem Anwachsen der Städte während der ersten Hälfte des 20. Jahrhunderts hat die Kapitalentwertung in Innenstadtquartieren aus dem 19. Jahrhundert die Bedingungen geschaffen, unter denen lukrative Neuinvestitionen möglich sind. Wenn diese Ertragslückentheorie der Gentrifizierung zutrifft, dann wäre davon auszugehen, dass Sanierung dort ihren Ausgang genommen hat, wo die Lücke am größten war und die höchste Rendite winkte, das heißt in besonders innenstadtnahen Quartieren und da, wo das Ende der Wertverlustkette bereits mehr oder weniger erreicht war. Empirisch scheint dies der Fall gewesen zu sein. Die Theorie legt auch den Schluss nahe, dass nach Sanierung dieser ersten Gebiete andere Gegenden ausfindig gemacht werden, die zwar geringere, aber immer noch ordentliche Rendite versprechen. Dies beträfe Quartiere, die weiter vom Stadtzentrum entfernt sind beziehungsweise deren Niedergang noch nicht so weit fortgeschritten ist. In Philadelphia wurden folglich die Viertel Fairmount und Queen Village zu den neuen ,Hotspots' (Cybriwsky 1978; Levy 1978) und die von der Stadt verfolgte Auswahlstrategie für die Zuweisung von Bundesmitteln lässt vermuten, dass Teile von North Philadelphia die nächsten Sanierungskandidaten sein werden.

Erwähnenswert ist die Rolle des Staates bei früheren Sanierungsprogrammen. Indem er Immobilien zum Verkehrswert zusammentrug und den Developern zum niedrigeren Schätzpreis überließ, vollzog der Staat die letzte Phase der Kapitalentwertung und übernahm die Kosten dafür. Dadurch gewährleistete er, dass die Developer jene hohen Renditen einstreichen konnten, ohne die keine Sanierung durchgeführt würde. Heute, wo der Staat weniger aktiv in den Prozess involviert ist, sind die Developer definitiv in der Lage, die Kosten der Entwertung noch nicht vollständig wertgeminderten Kapitals selbst zu tragen. Das heißt, sie können relativ hohe Preise für zu sanierende Immobilien bezahlen und erzielen immer noch eine ordentliche Rendite. Dem Staat scheint es also gelungen zu sein, die Rahmenbedingungen zu schaffen, die eine privatwirtschaftlich getragene Stadterneuerung ankurbeln.

Zusammengefasst besagt die Ertragslückentheorie, dass Gentrifizierung ein strukturelles Produkt des Grundstücks- und Wohnungsmarktes ist. 
Kapital fließt dorthin, wo die Investition am rentabelsten ist, und der Abfluss von Kapital in den suburbanen Raum erzeugt zusammen mit dem fortlaufenden Wertverlust innerstädtischen Kapitals letzten Endes die Ertragslücke. Ist diese Lücke ausreichend groß geworden, kann Sanierung (und übrigens auch Umbau) mit den anderswo erzielbaren Renditen konkurrieren und das Kapital fließt zurück.

\section{Fazit}

Entgegen der herkömmlichen Auffassung zeigt Gentrifizierung, dass die Wohnformen der Mittel-und Oberschicht auch mit intensiver Flächennutzung einhergehen können. Wie intensiv, das ist allerdings noch unklar. Es gibt deutliche Belege dafür, dass die einst steil ansteigende Bodenwertkurve (siehe Abb. 1) abflacht (Yeates 1965; Edel/Sclar 1975), und wenn dem so ist, mag die potenzielle Grundrente in innerstädtischen Quartieren tatsächlich gefallen sein, vermutlich aufgrund effizienter Verkehrsanbindungen an den suburbanen Raum und wegen der maßlos überfüllten Stadtzentren. Wie sich dies auf Gentrifizierung auswirken könnte oder auf die Neuansiedlung von Gewerbe und Freizeitangeboten, die in manchen Städten ebenfalls zu beobachten ist, sollte Gegenstand weiterer Forschung sein. Eine andere empirisch zu untersuchende Frage ist, in welchem Umfang die Kapitalentwertung in einer Gegend vorangeschritten sein muss, bevor es zu Gentrifizierung kommen kann. All das beruht auf der Annahme, die Ertragslücke ergebe sich primär aus dem Sickereffekt, und während dies in den USA sicherlich zutrifft, mag dem anderswo nicht so sein. Obgleich Kapitalentwertung und Sickereffekt der Gentrifizierung im Londoner Stadtteil Islington den Weg ebneten (Pitt 1977), ist nicht davon auszugehen, dass dies für den britischen Wohnungsmarkt, wo Wohnraum für die Arbeiterschicht viel eher in kommunaler als in privater Regie geschaffen wird, insgesamt gilt. In diesem Falle könnte es sein, dass die infolge von Stadterweiterung und -entwicklung steigenden Grundrenten weit ausschlaggebender sind, um die Entstehung der Ertragslücke zu erklären.

Gentrifizierung ist in der Tat eine Bewegung zurück in die Innenstadt, aber eher eine des Kapitals als eine der Menschen. Bislang stammen diejenigen, die sich diesen Kapitalrückfluss zunutze machen, immer noch aus der Stadt selbst. Wenn die Stadt weiterhin Produktivkapital anzieht (sei es für Wohnungsbau oder für andere Bauvorhaben), dann könnten wir zu Zeugen einer grundlegenden, der Suburbanisierung vergleichbaren Umgestaltung des städtischen Raums werden. Dann hätten wir es tatsächlich auch mit einer Bewegung von Menschen zurück in die Innenstadt zu tun - das heißt, mit Angehörigen der Mittel- und Oberschicht, die wieder in die Stadt ziehen, während Arbeiterschicht und Arme in grausam ironischer Fortsetzung des Sickereffekts die alten, verfallenden suburbs übernehmen. Sie säßen dann nicht mehr in der Innenstadt fest, sondern in suburbia. Wie schon in der Diskussion um Suburbanisierung hervorgehoben wurde, sind Investitionen in die gebaute Umwelt ein wesentliches Mittel zur Kapitalakkumulation. Dieser Prozess ist zyklischer Natur und neue Investitionszyklen gehen aufgrund der langen Lebensdauer und Gebundenheit solcher Investitionen häufig mit Krisen und einer Verlagerung des Schauplatzes der Akkumulation 
einher (Harvey 1978). In diesem Kontext gesehen könnten Gentrifizierung und andere Formen der urbanen Renaissance die Vorboten (aber keinesfalls die Ursache) einer größeren räumlichen Umstrukturierung darstellen. Ein Szenario besagt, diese Umstrukturierung werde gemäß den Bedürfnissen des Kapitals erfolgen - eine Neuausrichtung der Mittelschichtkultur könne zwar mit ihr einhergehen und sie beeinflussen, sei aber zweitrangig. Einem anderen Szenario zufolge könnten die Bedürfnisse des Kapitals systematisch außer Kraft gesetzt und als Organisationsprinzip der räumlichen Umstrukturierung von den sozialen, ökonomischen und kulturellen Bedürfnissen der Menschen abgelöst werden.

Anmerkung des Autors: Eine frühere Version dieses Beitrags wurde am 25. April 1979 auf der Jahreskonferenz der Association of American Geographers in Philadelphia vorgestellt. Mein besonderer Dank gilt Michele LeFaivre für die kritische Lektüre des Artikels.

\section{Übersetzung aus dem Englischen von Andrea Tönjes und Stephan Elkins für SocioTrans - Social Science Translation \& Editing}

\section{Endnoten}

[1] Der Text erschien 1979 im englischen Original „Toward a Theory of Gentrification. A Back to the City Movement by Capital, not People "im Journal of the American Planning Association (45, 4: 538-548). Wir danken dem Journal of the American Planning Association für die freundliche Genehmigung für die Veröffentlichung der deutschen Übersetzung.

[2] Gentrifizierung bezeichnet einen Prozess, im Zuge dessen Arbeiterviertel mittels Sanierung des örtlichen Wohnungsbestandes in Mittelschichtsquartiere umgewandelt werden.

[3] Um den Originaltext möglichst unverändert wiederzugeben bzw. Schwierigkeiten in der Übersetzung des Textes zu vermeiden, wurde in der vorliegenden Übersetzung entgegen der sonstigen Politik der Zeitschrift auf geschlechtsneutrale bzw. -inklusive Formulierungen verzichtet (Anm. d. Red.).

[4] Alle Literaturzitate sind aus dem Englischen übersetzt (Anm. d. Übers.).

[5] Der Umstand, dass diese frühen Projekte erheblicher staatlicher Initiative und Subventionen bedurften, schloss nicht aus, sie unter Verweis auf Konsumentenpräferenzen zu erklären. In Philadelphia beispielsweise brachte die Organisation Greater Philadelphia Movement (GPM) (ein Zusammenschluss lokaler Unternehmen gegen Korruption und Misswirtschaft in der Stadtverwaltung, Anm. d. Übers.) die Politik dazu, den Stadterneuerungsplan für Society Hill umzusetzen, und behauptete stets, der Ruf nach Sanierung sei zwar allgegenwärtig, der finanzielle Aufwand und das Risiko für Privatkapital bzw. Privatpersonen aber zu hoch. Es liege in der Verantwortung des Staates, so ihr Argument, die geltenden Bundesgesetze anzuwenden, um das Projekt zu subventionieren und dadurch Hindernisse aus dem Weg zu räumen. Dies diene dem Gemeinwohl der Gesellschaft insgesamt. Zur Rolle des GPM in Society Hill siehe Adde (1969: 33 ff.). Für diesen Beitrag unterscheide ich zwischen Gentrifizierung und Stadterneuerung nicht anhand der Frage, ob der Prozess öffentlich oder privat finanziert ist, sondern anhand dessen, ob es sich um Sanierungsmaßnahmen oder rein um Neubau handelt. Wie aus dem Hauptargument des Artikels deutlich werden sollte, stellen öffentliche und private Finanzierung (in diesem Kontext) lediglich zwei unterschiedliche Mechanismen zur Durchführung des einen maßgeblichen Prozesses dar.

[6] Das Ribicoff-Komitee war ein Senatsausschuss unter Vorsitz des demokratischen Senators Abraham A. Ribicoff (Anmerkung d. Übers.). 
[7] Als suburbaner Raum bzw. suburbia sind hier Gebiete bezeichnet, die außerhalb der gegenwärtigen Stadtgrenzen, aber innerhalb des statistisch definierten Ballungsgebietes (SMSA - Standard Metropolitan Statistical Area; Anm. d. Übers.) liegen. Die älteren Vororte, die infolge von Eingemeindung heute innerhalb der Stadtgrenzen liegen, werden daher als Teile der Stadt behandelt. Diese Definition ist hier gerechtfertigt, weil eines der Hauptargumente, die für Gentrifizierung ins Feld geführt werden, lautet, dass sie der Stadt zusätzliche Steuereinnahmen bringe. Ganz offensichtlich fließen die Steuern aus den eingemeindeten Vororten ja bereits an die Stadt.

[8] Diese Art der Konsolidierung mag sich (auch; Anm. d. Übers.) in anderen Städten gezeigt haben. Einige der von Lipton (1977) untersuchten Städte wiesen eine ähnliche Konsolidierung auf.

[9] Für die weitere Diskussion im transatlantischen Vergleich siehe Smith (1979).

[10] Werbung ist ein elementares Mittel um Nachfrage zu erzeugen. In Society Hill hat die Old Philadelphia Development Corporation einen Madison Avenue Fachmann engagiert um das Projekt zu bewerben (Old Philadelphia Development Corporation 1970).

[11] Natürlich setzt der Staat der Verfügungsgewalt der Eigentümer über ihren Grund und Boden merkliche Grenzen (durch Flächennutzungspläne, Enteignung für öffentliche Zwecke und andere staatliche Regulierungen), aber in Nordamerika und Europa sind diese Einschränkungen kaum mehr als kosmetischer Natur. Innerhalb dieser Grenzen funktioniert der Immobilienmarkt weiterhin recht ungehindert.

[12] ,The Loop“ ist die lokal gebräuchliche Bezeichnung für das zentrale Geschäftsviertel der Chicagoer Innenstadt (Anmerkung d. Übers.).

[13] Beim redlining werden Quartiere tatsächlich auf dem Stadtplan rot umrandet (Anmerkung d. Übers.).

[14] Die Federal Housing Administration ist eine nachgeordnete Behörde des U.S. Department of Housing and Urban Development, die Gelder für Hausbau und Erwerb zur Verfügung stellt. Das im Zuge des New Deal 1934 eingeführte Programm soll es auch Haushalten mit geringerem Einkommen ermöglichen, ein Eigenheim zu besitzen (Anmerkung d. Übers.).

[15] Die Annahme eines Sickereffekts ist in heutigen Debatten zu Wohnungsmarkt und -politik weit verbreitet. Neoklassische Ökonomen nehmen an, dass die Bereitstellung teurer Wohnungen im oberen Preissegment auch die Versorgung von einkommensschwachen Haushalten verbessert. Durch das zusätzliche Angebot teurer Wohnungen soll der Preis in den bisher teuersten Segmenten des Markts sinken und damit der positive Effekt der Angebotsausweitung durch den Markt, sickern'. Dieser Theorieansatz wird von der kritischen Wohnungsforschung seit langem stark kritisiert (siehe etwa Krätke, Stefan (1995): Stadt - Raum - Ökonomie: Einführung in Aktuelle Problemfelder der Stadtökonomie und Wirtschaftsgeographie. Basel: Birkhäuser Verlag). Smith bezieht sich in seiner Ausführung auf Lowry (1960), der eine sehr spezifische Definition des Sickereffekts ausführt. Er bezeichnet ihn als die veränderte, relative Position einer Wohnungseinheit in Bezug auf ihre Miete bzw. ihren Preis in der Gesamtverteilung aller Wohneinheiten in einer Nachbarschaft. (Anm. d. Red.)

[16] Unter ,kollektivem sozialen Handeln“ verstehe ich schlicht Handlungen, die nicht vom Einzelnen allein, sondern zusammenwirkend und gleichzeitig vollzogen werden.

[17] Dass ich Spekulanten nicht berücksichtigt habe, hat den offensichtlichen Grund, dass sie kein produktives Kapital investieren. Sie kaufen lediglich Immobilien in der Hoffnung, sie zu einem höheren Preis an Developer weiterverkaufen zu können. Spekulanten tragen somit nicht zur urbanen Umstrukturierung bei.

\section{Autor_innen}

Neil Smith war Stadtgeograph und forschte unter anderem zu ungleicher Entwicklung, zur Sozialkonstruktion von Natur und zu Gentrifizierungsprozessen. 


\section{Literatur}

Adde, Leo (1969): Nine cities: anatomy of downtown renewal. Washington D.C.: Urban Land Institute.

Alonso, William (1964): Location and land use. Cambridge: Harvard University Press.

Baltimore City Department of Housing and Community Development (1977): Homesteading - the third year, 1976. Baltimore: Department of Housing and Community Development.

Bradford, Calvin P. / Rubinowitz, Leonard S. (1975): The urban-suburban investment-disinvestment process: consequences for older neighborhoods. In: Annals of the American Academy of Political and Social Science 422, 77-86.

Burt, Nathaniel (1963): The perennial Philadelphians. London: Dent \& Son.

Cybriwsky, Roman A. (1978): Social aspects of neighborhood change. In: Annals of the Association of American Geographers 68, 17-33.

Davis, J. Tait (1965): Middle class housing in the central city. In: Economic Geography 41, 238-251.

Edel, Matthew / Sclar, Elliot (1975): The distribution of real estate value changes: metropolitan Boston, 1870-1970. In: Journal of Urban Economics 2, 366-387.

Gale, Dennis E. (1976): The back-to-the-city movement... or is it? Occasional Paper, Department of Urban and Regional Planning, George Washington University.

Gale, Dennis E. (1977): The back-to-the-city movement revisited. Occasional Paper, Department of Urban and Regional Planning, George Washington University.

Gans, Herbert (1968): People and plans. New York: Basic Books.

Greenfield, A. M. and Co. Inc. (1964): New town houses for Washington Square East: a technical report on neighborhood conservation. Bericht erstellt für die Redevelopment Authority of Philadelphia.

Harvey, David (1973): Social justice and the city. Baltimore: Johns Hopkins Press.

Harvey, David (1978): The urban process under capitalism: a framework for analysis. In: International Journal of Urban and Regional Research 2, 101-131.

Harvey, David / Baltimore Urban Observatory (1972): The housing market and code enforcement in Baltimore. Baltimore: City Planning Department.

Hoyt, Homer (1933): One hundred years of land values in Chicago. Chicago: University of Chicago Press.

Laurenti, Luigi (1960): Property values and race. Berkeley: University of California Press.

Levy, Paul R. (1978): Queen Village: the eclipse of community. Philadelphia: Institute for the Study of Civic Values.

Ley, David (1978): Inner city resurgence and its societal context. Vortrag gehalten auf der Jahreskonferenz der Association of American Geographers in New Orleans.

Lipton, S. Gregory (1977): Evidence of central city revival. In: Journal of the American Institute of Planners 43, 136-147.

Lowry, Ira S. (1960): Filtering and housing costs [sic!]: a conceptual analysis. In: Land Economics 36, 362-370.

McDonald, John F. / Bowman, H. Wood (1979): Land value functions: a reevaluation. In: Journal of Urban Economics 6, 25-41.

Mills, Edwin S. (1972): Studies in the structure of the urban economy. Baltimore: Johns Hopkins University Press.

Muth, Richard F. (1969): Cities and housing. Chicago: University of Chicago Press.

Old Philadelphia Development Corporation (1975): Statistics on Society Hill. Unveröffentlichter Bericht.

Pitt, James (1977): Gentrification in Islington. London: Barnsbury Peoples Forum.

Smith, Neil (1979): Gentrification and capital: theory, practice and ideology in Society Hill. In: Antipode 11, 163-173.

Stegman, Michael A. (1972): Housing investment in the inner city: the dynamics of decline. Cambridge: MIT Press.

Urban Land Institute (1976): New opportunities for residential development in central cities. Report No. 25. Washington D.C.: Urban Land Institute.

Winters, Christopher (1978): Rejuvenation with character. Vortrag gehalten auf der Jahreskonferenz der Association of American Geographers in New Orleans. 
Wolf, Edwin II. (1975): Philadelphia: portrait of an American city. Harrisburg: Stackpole Books.

Yeates, Maurice H. (1965): Some factors affecting the spatial distribution of Chicago land values, 1910-1960. In: Economic Geography 41/1, 57-70.

\section{Toward a Theory of Gentrification. A Back to the City Move- ment by Capital, not People}

Consumer sovereignty hypotheses dominate explanations of gentrification but data on the number of suburbanites returning to the city casts doubt on this hypothesis. In fact, gentrification is an expected product of the relatively unhampered operation of the land and housing markets. The economic depreciation of capital invested in nineteenth century innercity neighborhoods and the simultaneous rise in potential ground rent levels produces the possibility of profitable redevelopment. Although the very apparent social characteristics of deteriorated neighborhoods would discourage redevelopment, the hidden economic characteristics may well be favorable. Whether gentrification is a fundamental restructuring of urban space depends not on where new inhabitants come from but on how much productive capital returns to the area from the suburbs. 